\title{
USABILIDADE DE PRODUTOS DE TECNOLOGIA ASSISTIVA PARA ATIVIDADES DE VIDA DIÁRIA DE PESSOAS COM DOENÇA DE PARKINSON
}

\section{USABILITY OF ASSISTIVE TECHNOLOGY PRODUCTS FOR DAILY LIVING ACTIVITIES OF PEOPLE WITH PARKINSON DISEASE}

Ana Karina Pessoa da Silva Cabral ${ }^{1}$, M.Sc.

Danielle Carneiro de Menezes Sanguinetti ${ }^{2}$, D.Sc.

Daniela Salgado $\mathrm{Amaral}^{3}$, M.Sc.

Juliana Fonsêca de Queiroz Marcelino ${ }^{4}$, M.Sc.

Laura Bezerra Martins ${ }^{5}$, Ph.D.

(1) Universidade Federal de Pernambuco

e-mail: anakarina.ufpe@gmail.com

(2) Universidade Federal de Pernambuco e-mail:dcmsanguinetti@gmail.com

(3) Universidade Federal de Pernambuco e-mail:_danisamaral@hotmail.com

(4) Universidade Federal de Pernambuco e-mail: julifons@yahoo.com.br

(5) Universidade Federal de Pernambuco e-mail: bmartins.laura@gmail.com

Palavras-chave (Usabilidade, Ergonomia, Tecnologia Assistiva)

\begin{abstract}
A Doença de Parkinson (DP) é uma das principais causas de incapacidades físicas em pessoas acima de 60 anos. Para facilitar a realização das atividades de vida diária podem ser usados produtos de Tecnologia Assistiva, como as adaptações. O objetivo da pesquisa foi avaliar a usabilidade de produtos de Tecnologia Assistiva, impressos em 3D, para atividades de vida diária de pacientes com Doença de Parkinson, considerando as variáveis ligadas ao usuário e ao desempenho da tarefa (eficiência, eficácia e satisfação). Realizou-se estudo de campo, descritivo, com 5 sujeitos, por meio de entrevistas, observações do desempenho e aplicação de avaliação de satisfação do usuário com Tecnologia Assistiva de Quebec B-QUEST (2.0). Os resultados permitiram estabelecer requisitos para o projeto de produtos (adaptações) impressos em 3D, para serem usados nas atividades cotidianas de pessoas com DP, favorecendo mais autonomia e independência. Recomenda-se estudos futuros acerca da propriedade dos materiais das adaptações e do desempenho no ambiente cotidiano das pessoas com DP.
\end{abstract}




\section{$16^{\circ}$ \\ ERGODESIGN USIHC CINAHPA}

$16^{\circ}$ Ergodesign - Congresso Internacional de Ergonomia e Usabilidade de Interfaces Humano Tecnológica: Produto, Informações Ambientes Construídos e Transporte

$16^{\circ}$ USIHC - Congresso Internacional de Ergonomia e Usabilidade de Interfaces Humano Computador

CINAHPA | 2017 - Congresso Internacional de Ambientes Hipermídia para Aprendizagem.

Key-words (Usability, Ergonomics, Assistive Technology)

Parkinson's disease (PD) is one of the leading causes of physical disability in people over 60 years of age. Assistive Technology products such as adaptations can be used to facilitate the activities of daily living. The objective of the research was to evaluate the usability of $3 D$ Assistive Technology products for daily activities of patients with Parkinson's disease, considering the variables related to the user and the performance of the task (efficiency, effectiveness and satisfaction). A descriptive field study was carried out with 5 subjects, through interviews, performance observations and application of user satisfaction evaluation with the Quebec Assistive Technology BQUEST (2.0). The results allowed to establish requirements for the design of products (adaptations) printed in 3D, to be used in the daily activities of people with PD, favoring more autonomy and independence. Future studies on the ownership of adaptation materials and performance in the daily environment of people with $P D$ are recommended.

\section{Introdução}

De acordo com o censo de 2010 do Instituto Brasileiro de Geografia e Estatísticas (IBGE), 7\% da população possui algum tipo de deficiência física. A mesma pesquisa aponta a Região Nordeste com a maior prevalência de deficiências (OLIVEIRA, 2012).

Dentre as causas de deficiência está a Doença de Parkinson (DP) que é considerada a segunda doença neurodegenerativa mais incidente no mundo. Sua degeneração está ligada aos gânglios de base e se apresenta essencialmente por disfunções motoras.

É uma das principais causas de incapacidades físicas em pessoas acima de 60 anos. Essa doença reduz a produção do neurotransmissor dopamina que desencadeia uma sensação de fadiga, tremores de caráter progressivo, evoluindo para graus de rigidez muscular, bradicinesia e alterações de postura e instabilidade (ALMEIDA; CRUZ, 2009; SILVA et al, 2010).

Os principais sintomas da doença acarretam dificuldades na realização das Atividades de Vida Diária (AVD). Em fases mais avançadas, aparecem distúrbios motores significativos, fazendo o indivíduo necessitar de ajuda para realizar algumas ou todas as suas AVD (ALMEIDA; CRUZ, 2009; SILVA et al, 2010).

Terapeutas ocupacionais, com o intuito de estimular a funcionalidade, usam produtos de Tecnologia Assistiva, como adaptadores de botões, engrossadores de talher, pratos com bordas elevadas, barras de apoio, entre outros, para facilitar a realização das atividades, favorecendo mais independência ao indivíduo (PELOSI, 2005).

Apesar dos avanços tecnológicos e da existência de equipamentos de Tecnologia Assistiva para esse fim, o uso da impressora 3D para confecção de adaptações para pacientes com disfunções físicas é inovador no Brasil.

Esse equipamento pode possibilitar o desenvolvimento de adaptações mais adequadas ao paciente, permitindo melhor ajuste, sem sobreposições e costuras, além de ser mais durável e de baixo custo, com relação aos que existem no mercado.

Para confeccionar uma adaptação deve-se considerar a simplicidade do projeto, o custo, o usuário, conforto, estética, higiene e integridade dos tecidos moles.

O objetivo do estudo foi avaliar a usabilidade de produtos de Tecnologia Assistiva, impressos em 3D, para atividades de vida diária de pacientes com Doença de Parkinson, considerando as variáveis ligadas ao usuário e ao desempenho da tarefa (eficiência, eficácia e satisfação).

\subsection{Desempenho Ocupacional na Doença de Parkinson e Tecnologia Assistiva}

De acordo com a Associação Americana de Terapia Ocupacional (AOTA, 2010), as áreas de ocupação

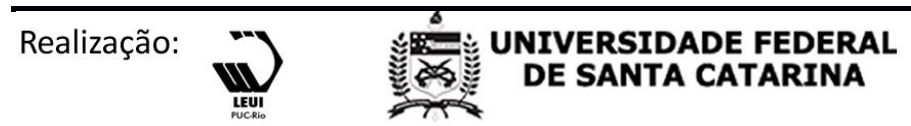




\section{$16^{\circ}$ \\ ERGODESIGN USIHC CINAHPA}

$16^{\circ}$ Ergodesign - Congresso Internacional de Ergonomia e Usabilidade de Interfaces Humano Tecnológica: Produto, Informações Ambientes Construídos e Transporte

$16^{\circ}$ USIHC - Congresso Internacional de Ergonomia e Usabilidade de Interfaces Humano Computador

CINAHPA | 2017 - Congresso Internacional de Ambientes Hipermídia para Aprendizagem. correspondem às atividades cotidianas nas quais as pessoas, populações ou organização se envolvem, incluem AVD, Atividades Instrumentais de Vida Diária (AIVD), descanso e dormir, educação, trabalho, brincar, lazer e participação social.

As AVD são atividades voltadas para o cuidado pessoal do individuo, tais como tomar banho, vestir-se, comer, mover-se de um local para o outro (mobilidade funcional). Já as AIVD são tarefas mais complexas realizadas dentro de casa ou na comunidade, como usar meios de transporte, preparar refeições, fazer tarefas domésticas, fazer compras, cuidar da casa (CARLETO; et al, 2010).

As disfunções que podem ocorrer nas AVD são denominadas de incapacidades, segundo o modelo de incapacidade desenvolvido pela Organização Mundial de Saúde (ROGERS; HOLM, 2002). O declínio que ocorre nas AVD dos indivíduos com DP é ocasionado pelos comprometimentos motores da doença, que geram uma redução no nível de funcionalidade, de acordo com a progressão da doença (SOUZA; et al, 2007; FINDLEY, 2007).

No estudo realizado por Souza et al (2007) foi evidenciado que, pessoas com DP com mais de 5 anos de evolução da doença apresentaram piores escores na função das AVD, se comparados a pacientes com 5 anos ou menos de DP.

Souza et al (2011) e Jankovic (2007) referem que a pobreza dos movimentos e a lentificação, tanto na iniciação como na execução de atos voluntários e involuntários, levam os indivíduos com DP a demandar mais tempo e esforço para realizarem as atividades rotineiras, como tomar banho, vestir e pagar as contas.

De acordo com Cavalcanti e Galvão (2007), os dispositivos de tecnologia objetivam promover segurança, conforto, facilidade e ampliação do desempenho e funcionalidade do sujeito.

Bersh (2013) acrescenta que os dispositivos para auxílios nas AVD e AIVD favorecem o desempenho autônomo e independente, facilitando o cuidado de pessoas com limitação, em atividades como se alimentar, cozinhar, vestir-se e tomar banho.

Alguns equipamentos assistivos são indicados para pessoas com DP com intuito de promover a capacidade funcional e proporcionar maior autonomia nas atividades, como o uso de adesivos ou antiderrapantes e barras de apoio para favorecer segurança, adaptadores de botão, talheres engrossados com peso para diminuir movimentos involuntários, prato com borda e ventosa, copo recortado com alça e caneta engrossada (SANTOS; RUIZ; FRANCISCO, 2006).

\subsection{Ergonomia e Usabilidade}

A Usabilidade, de acordo com o próprio sentido da palavra, foca-se em como as pessoas usam o produto, no que concerne a interação entre usuário, a tarefa e o produto.

Durante a década de 90, com a saturação do mercado e a queda das diferenças tecnológicas, aspectos como a estética e a usabilidade passaram a ter mais importância, os parâmetros de usabilidade receberam maior atenção, inicialmente com foco nos estudos de mercado, envolvendo cada vez mais o usuário no processo de design (FALCÃO; SOARES, 2013).

No Brasil, a usabilidade é definida, segundo a NBR 9241-11/2011, como a medida na qual um produto pode ser usado por usuários específicos para alcançar objetivos específicos com eficácia, eficiência e satisfação, em um contexto específico de uso (ABNT, 2011).

Considera-se ainda a aplicação da ISO 9241-11 (1998), que formula recomendações para a mensuração das qualidades ergonômicas do produto, levando em conta os mesmos fatores de qualidade: eficácia, eficiência e a satisfação.

Para o melhor entendimento desta última definição, Jordan (1998) esclarece:

- Eficácia: se refere à extensão na qual uma meta é alcançada ou uma tarefa é realizada.

- Eficiência: se refere à quantidade de esforço requerido para se atingir uma meta. Quanto menos
Realização:

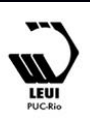


esforço, maior é a eficiência.

- Satisfação: se refere ao nível de conforto que os usuários sentem quando utilizam um produto e também ao nível de aceitação do produto pelos mesmos para atingir suas metas.

De acordo com Tullis e Albert (2008), medir a experiência do usuário oferece muito mais do que apenas uma simples observação. Métricas adicionam estrutura ao processo de concepção e avaliação, indicando os resultados e fornecendo informações para a tomada de decisões.

As métricas utilizadas para a avaliação objetiva da usabilidade de um produto, de acordo com Jordan (1998), relacionam-se com os três aspectos da usabilidade citados anteriormente:

- Métricas de eficácia: completeza da tarefa; qualidade do resultado.

- Métricas de eficiência: desvios do caminho crítico (o mais eficiente para realizar a tarefa); quantidade ou taxa de erros; tempo para a realização da tarefa; carga mental (medida de diferentes formas).

- Métricas de satisfação: análise qualitativa da satisfação (por meios de entrevistas e questionários, por exemplo); análise quantitativa da satisfação, por meio de escala quantitativa, como de Likert (1932).

No processo de avaliação da usabilidade, propriamente dito, são utilizados Métodos para a Avaliação dirigidos a Especialistas e a Usuários avaliações de desempenho (quantitativas) e (qualitativas). Para Jordan (1998), não há nada que substitua a possibilidade de ver o usuário tentando utilizar o produto.

Sobre a escolha dos usuários a serem observados, Nielsen (1993) sugere que ocorra da maneira mais variada possível, considerando repertório, localização, estilo de vida, entre outros quesitos, com o intuito de captar os diversos panoramas da percepção do produto.

Uma vantagem proporcionada por essa técnica é $16^{\circ}$ Ergodesign - Congresso Internacional de Ergonomia e Usabilidade de Interfaces Humano Tecnológica: Produto, Informações Ambientes Construídos e Transporte

$16^{\circ}$ USIHC - Congresso Internacional de Ergonomia e Usabilidade de Interfaces Humano Computador

CINAHPA | 2017 - Congresso Internacional de Ambientes Hipermídia para Aprendizagem.

que o usuário se encontra em seu ambiente natural, portanto, as posturas assumidas por ele serão mais verdadeiras e fiéis do que as interações visualizadas em laboratório.

No entanto, as observações de campo acarretam a desvantagem de uma exigência maior do tempo, devido a fatores sob os quais o observador não possui controle, como interrupções e ruídos do sistema.

\section{Procedimentos Metodológicos}

\subsection{Desenho do estudo}

A pesquisa consiste em um estudo de campo, descritivo, observacional, de corte transversal. Para Nielsen (1993), o estudo de campo é uma técnica de grande importância no desenvolvimento do sistema/produto, pois analisa o usuário e suas interações com o sistema/produto, observando detalhadamente, de forma direta ou indireta, vários aspectos que somente são identificados no ambiente e contexto do usuário, como suas necessidades, fazendo anotações e perguntas aos usuários.

Para avaliação da usabilidade foi utilizado um Método Comparativo, no qual realizou-se a avaliação por Especialistas, em seguida por Usuários - avaliações de desempenho (quantitativas) e (qualitativas), finalizando com a comparação entre ambas.

\subsection{Sujeitos da pesquisa}

A população do estudo são pessoas com Doença de Parkinson (DP) cadastradas no Programa de Extensão Pró-Parkinson, do Hospital das Clínicas da Universidade Federal de Pernambuco (HC/UFPE).

A amostra foi selecionada de forma intencional e não-probabilística, sendo constituída por 05 (cinco) indivíduos com DP, atendidos no Ambulatório de Neurologia do HC/UFPE.

Os critérios de inclusão foram: pessoas com diagnóstico de DP idiopática, adultos e idosos, de
Realização:

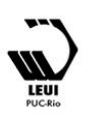




\section{$16^{\circ}$ \\ ERGODESIGN USIHC CINAHPA}

ambos os sexos, sem limite de faixa etária e escolaridade, sem restrição quanto a classe social, tempo de diagnóstico e profissão, pontuação acima de 18 (dezoito) no Mini Exame do Estado Mental (MEEM).

O MEEM é o teste de rastreio cognitivo mais utilizado no mundo (FOSTEIN et al. 1975). Os valores mais altos do escore indicam maior desempenho cognitivo.

Os critérios de exclusão foram: pessoas com diagnóstico de demência ou doença psiquiátrica; que apresentem qualquer outra doença neurológica, doenças sistêmicas descompensadas e/ou doenças ortopédicas e reumatológicas; que não possuam necessidade de utilizar o produto de TA para melhorar seu desempenho nas atividades cotidianas (aspecto identificado na primeira estapa do estudo).

\subsection{Coleta dos dados}

Os dados foram coletados no período de fevereiro a março de 2017, por meio da Escala Unificada de Avaliação para Doença de Parkinson (UPDRS) e a Medida Canadense de Desempenho Ocupacional (COPM).

A Escala Unificada (UPDRS) avalia os sinais, sintomas e determinadas atividades dos pacientes (MARTIGNONI et al, 2003). É composta por 42 itens, divididos em quatro partes: atividade mental, comportamento e humor; atividades de vida diária (AVD's); exploração motora e complicações da terapia medicamentosa. Nesse estudo foi usada a seção II de AVD que identificou atividades comprometidas e intensidade .

A Medida Canadense de Desempenho Ocupacional (COPM) avaliou mudanças na percepção do indivíduo sobre seu desempenho nas atividades, bem como mudanças em seu nível de satisfação em relação a esse desempenho. Essas atividades podem enquadrar-se em qualquer uma das áreas de desempenho: auto-cuidado, trabalho e lazer.

(POLLLOCK et al., 2003).

As avaliações supracitadas foram aplicadas com o $16^{\circ}$ Ergodesign - Congresso Internacional de Ergonomia e Usabilidade de Interfaces Humano Tecnológica: Produto, Informações Ambientes Construídos e Transporte

$16^{\circ}$ USIHC - Congresso Internacional de Ergonomia e Usabilidade de Interfaces Humano Computador

CINAHPA | 2017 - Congresso Internacional de Ambientes Hipermídia para Aprendizagem.

objetivo de identificar quais as atividades de vida diária que estavam mais comprometidas e em que grau de intensidade, para, assim, direcionar a seleção do artefato a ser desenvolvido, a partir da determinação do "conceito do produto".

Conforme Filho (2008), o conceito de um produto diz respeito ao conjunto de requisitos que devem ser observados, atendidos ou satisfeitos para que uma solução identificada possa ser considerada como viável. Corresponde a uma construção imaginária, no "plano imaterial". É o primeiro passo para a determinação das características materiais do produto.

Dentre as AVD mais citadas pelos 29 participantes da primeira etapa da pesquisa, ficou o vestir com $70 \%$ (14), seguido da alimentação 55\% (11), banho 25\% (5), mobilidade funcional $25 \%$ (5) autocuidado $20 \%$ (4).

Tendo em vista que o desempenho das atividades descritas envolve a necessidade do manuseio de utensílios diversos (talher, barbeador, lápis, escova de dentes), buscou-se desenvolver uma adaptação que se ajustasse ao máximo de conformações possível.

Em seguida, foi prescrita e confeccionada adaptação universal impressa em 3D para utensílios das atividades de vida diária comprometidas, tais como: adaptador de talher, de lápis e de barbeador, visando adequar o poduto a preensão manual do indivíduo.

Após 30 dias, foram aplicadas entrevistas informais que contemplaram as métricas de usabilidade, segundo Jordan (1998) : eficiência, eficácia e satisfação (conforto, segurança, desempenho da tarefa).

Considerou-se ainda os critérios de medição de usabilidade estabelecidos pela norma ISO 9241, como: análise das características requeridas do produto num contexto de uso específico, do processo de interação entre o usuário e o produto, da eficiência (agilidade na viabilização da tarefa), da eficácia (garantia da obtenção dos resultados 


\section{$16^{\circ}$ \\ ERGODESIGN USIHC CINAHPA}

desejados) e da satisfação resultante do uso desse produto.

Para mensurar a satisfação foi utilizada o instrumento de Avaliação do usuário com a Tecnologia Assistiva de Quebec (B-Quest), traduzido e validado no Brasil em 2014.

O B-QUEST (2.0) consiste em 12 itens de satisfação, 8 relacionados ao uso do recurso de Tecnologia assistiva, (dimensões, peso, ajustes, segurança, durabilidade, facilidade de uso, conforto, eficácia), alvo dessa pesquisa, e 4 relacionados aos serviços prestados (processo de entrega, reparos e assistência técnica, serviços profissionais, serviços de acompanhamento).

Cada item é pontuado usando uma escala de 5 pontos que varia entre insatisfeito, pouco satisfeito, mais ou menos satisfeito, bastante satisfeito e totalmente satisfeito (CARVALHO; JÚNIOR; SÁ, 2014).

De acordo com Carvalho (2013), O tempo para completar todos os itens da escala varia de 10 a 15 minutos. Um espaço para comentários é fornecido ao lado de cada item para identificar o motivo da insatisfação e a análise da média da pontuação do questionário se aplica pelas seguintes pontuações:

- 1 (ou muito próximo 1): Indica que os usuários estão "insatisfeitos" nesta sub escala;

- 2 (ou muito próximo de 2): Indica que os usuários estão " pouco satisfeitos" nesta sub- escala;

- 3 (ou muito próximo de 3): Indica que os usuários estão "mais ou menos satisfeitos" nesta sub- escala;

- 4 (ou muito próximo de 4): Indica que os usuários estão "bastante satisfeitos" nesta sub- escala;

- 5 (ou muito próximo de 5): Indica que os usuários estão "totalmente satisfeitos" nesta sub- escala

Realizou-se ainda análise da tarefa - realização da escrita e simulação do barbear, escovar dentes e alimentar-se, tendo em vista que os pacientes eram ambulatoriais, simulando o ambiente de uso próximo ao real, para distinguir em uma série de etapas os métodos para executar tarefas com o produto (LEVENTHAL; BARNES, 2008).

Essa técnica pode ser usada para revisões sobre como difícil ou fácil as tarefas serão realizadas e quanto esforço é provável que seja necessário. Buscou-se a identificação de possíveis soluções para os problemas de usabilidade. Para Jordan (1998), não há nada que substitua a possibilidade de ver o usuário tentando utilizar o produto.

Os dados quantitativos e qualitativos foram planilhados e organizados (Microsoft Excel e Word) e se aplicou a análise descritiva, por frequência simples e número de aparição.

\subsection{Aspectos éticos}

O projeto foi aprovado pelo Comitê de Ética em Pesquisa com Seres Humanos do Centro de Ciências da Saúde da UFPE, sob o registro CAAE 45871615.6.0000.5208, a partir da resolução 466/2012, do Conselho Nacional de Saúde.

Todos os participantes da pesquisa, assinaram o Termo de Consentimento Livre e Esclarecido (TCLE)

\section{Resultados}

Com relação aos principais aspectos sócio demográficos dos participantes da pesquisa (5 sujeitos), verificou-se 4 do sexo masculino, faixa etária entre 61 e 83 anos, com escolaridade entre ensino médio completo (2), incompleto (2) e analfabeto (1), diagnóstico Parkinson idiopático (doença primária, sem causa definida).

As atividades mais comprometidas citadas foram: alimentação, higiene, escrita e vestuário. Dessa forma, foi desenvolvida e impressa adaptação em 3D, universal, de modo a ser utilizada por esses pacientes nas diversas atividades de seu cotidiano (figuras 1, 2, 3 e 4). 


\section{$16^{\circ}$ \\ ERGODESIGN USIHC CINAHPA}

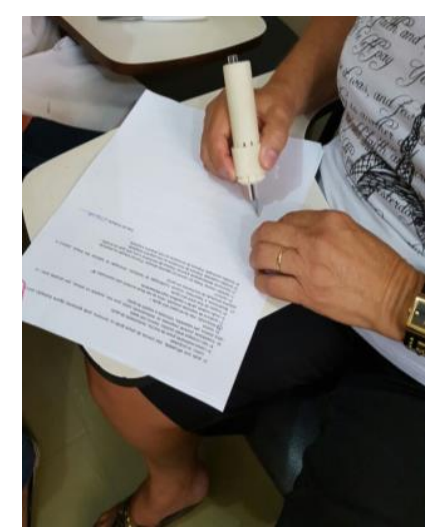

Fig. 1 - Adaptador universal usado na escrita (Fonte: autores)

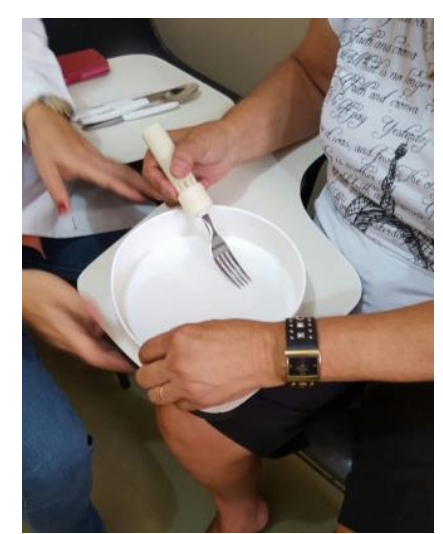

Fig. 2 - Adaptador universal usado na alimentação (Fonte: autores)

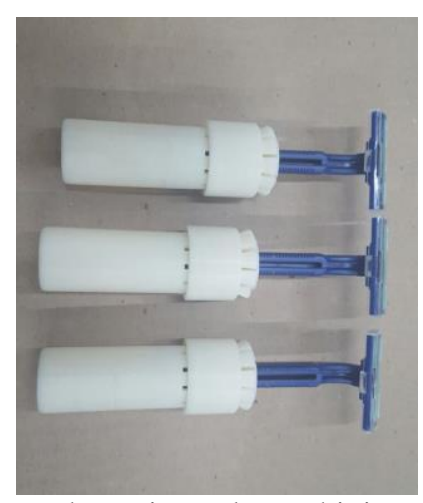

Fig.3 - Adaptador universal para higiene pessoal (Fonte: autores) $16^{\circ}$ Ergodesign - Congresso Internacional de Ergonomia e Usabilidade de Interfaces Humano Tecnológica: Produto, Informações Ambientes Construídos e Transporte

$16^{\circ}$ USIHC - Congresso Internacional de Ergonomia e Usabilidade de Interfaces Humano Computador

CINAHPA | 2017 - Congresso Internacional de Ambientes Hipermídia para Aprendizagem.

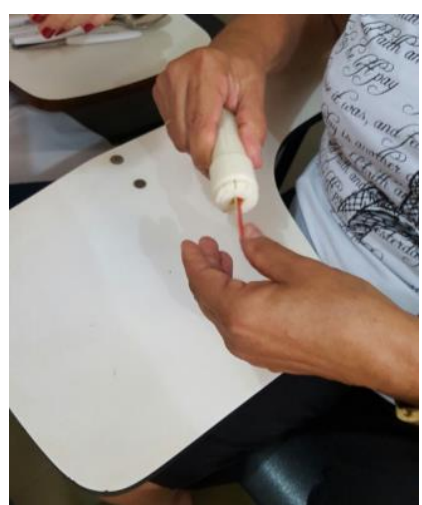

Fig.4 - Adaptador universal para higiene pessoal (Fonte: autores)

Sobre os atributos de usabilidade relacionados à satisfação com o produto (adaptador universal), o gráfico abaixo ilustra o relato dos usuários.

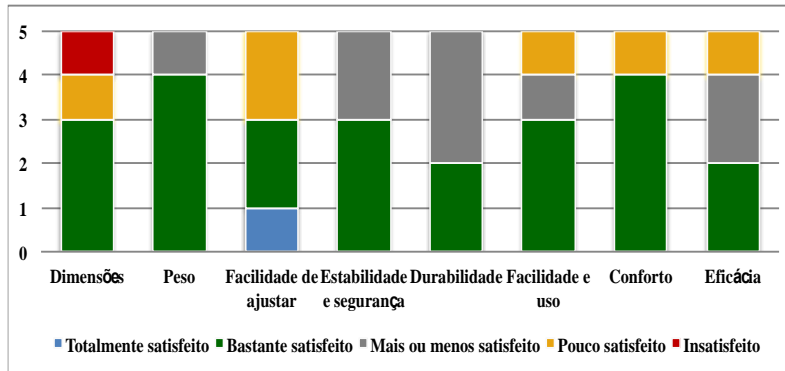

Gráfico - Avaliação do grau de satisfação com o adaptador universal.

A maioria dos usuários (3) afirmou estar bastante satisfeito com as dimensões (tamanho, altura, comprimento, largura) do produto de TA, com o peso (4), facilidade de ajustar (fixar) as partes do recurso (3), facilidade e uso do recurso (3), conforto (4), estabilidade e segurança do recurso (3).

As métricas com mais queixas foram:

- Durabilidade do produto (força e resistência ao desgaste), na qual 3 sujeitos relataram estar mais ou menos satisfeitos, devido a ruptura de componentes do produto, os demais sujeitos mencionaram bastante satisfação. 
$16^{\circ}$ Ergodesign - Congresso Internacional de Ergonomia e Usabilidade de Interfaces Humano Tecnológica: Produto, Informações Ambientes Construídos e Transporte

$16^{\circ}$ USIHC - Congresso Internacional de Ergonomia e Usabilidade de Interfaces Humano Computador

CINAHPA | 2017 - Congresso Internacional de Ambientes Hipermídia para Aprendizagem.
- Eficácia do produto (o quanto atende à necessidade), na qual 2 sujeitos afirmaram estar mais ou menos satisfeitos e 1 pouco satisfeito.
Quando questionados sobre os itens que consideram mais importantes, os 3 mais citados pelos entrevistados foram: peso, facilidade de uso, eficácia e ajustes. Dentre esses, a eficácia foi apontada como um item de insatisfação, conforme já descrito, apontando a necessidade de repensar a concepção do produto, com requisitos que garantam conforto, segurança, eficiência e satisfação dos usuários.

\subsection{Recomendações}

- O material utilizado na confecção da adaptação deve garantir mais resistência e durabilidade, e, ao mesmo tempo, favorecer a higienização e manutenção.

- O produto deve atender aos princípios do Design Universal, possibilitando o uso por maior número de usuários, mas buscando atender às necessidades clínicas diferentes.

- As dimensões da adaptação devem garantir adequação às medidas antropométricas do indivíduo, favorecendo conforto, diminuição de fadiga muscular e conservação de energia.

- A adaptação deve ajustar-se às dimensões dos utensílios usados no cotidiano dos indivíduos (talher, barbeador, caneta, entre outros).

\section{Considerações finais}

A pesquisa permitiu estabelecer requisitos para o aperfeiçoamento de adaptações impressas em 3D para pessoas com DP, visando independência no desempenho das AVD, levando-se em conta aspectos ergonômicos de usabilidade. No entanto, reconhecemos a limitação dos achados mediante o tamanho da amostra.

Pode-se afirmar que estudos de usabilidade do produto podem contribuir para identificar problemas no uso e definir requisitos para o desenvolvimento de produtos que atendam melhor às necessidades desses usuários.

Recomenda-se a realização da avaliação do desempenho do usuário em ambiente real, o que não foi possível nesse estudo, tendo em vista que os pacientes se encontravam em contexto ambulatorial. Nesse caso, foram realizadas simulações das atividades em ambientes adaptados para tal.

Como desdobramentos da pesquisa, apontamos ainda a necessidade de estudos sobre as propriedades dos materiais utilizados nas adaptações, quanto à resistência, durabilidade e baixo custo, de modo a garantir o aprimoramento do produto e difundir sua aplicação na rotina diária dessa população.

\section{Referências Bibliográficas}

ALMEIDA, M.; CRUZ, G. Intervenções de terapeutas ocupacionais junto a idosos com doença de Parkinson. Rev. Ter. Ocup. Univ, São Paulo, v. 20, n. 1, p.29-35, jan. 2009.

\section{BRYANT, D.; BRYANT, B. Assistive Technology for People with Disabilities. $2^{\text {a }}$ ed. 2012.}

CARVALHO, K. E. C.; JÚNIOR, M. B. G.; NUNES SÁ, K. Tradução e validação do Quebec User Evaluation of Satisfaction with Assistive Technology (QUEST 2.0) para o idioma português do Brasil. Rev. Bras Reumatol. v. 54, n. 4. p. 260267, 2014.

CARVALHO, K.E.C. de. Tradução para a Língua Portuguesa do Brasil e Validação do Quebec User Evaluation of Satisfaction with Assistive Technology (Quest 2.0). 2013. Tese (Mestrado em medicina) - Escola Bahiana de Medicina e Saúde Pública, Salvador, 2013.

CAVALCANTI, A.; GALVÃO, C. Terapia

Ocupacional: fundamentação e prática. Rio de Janeiro: Guanabara-Koogan, 2007. 531p.

Realização:




\section{$16^{\circ}$ \\ ERGODESIGN USIHC CINAHPA}

COOK, A.; POLGAR, J. Assistive Technologies: Principles and Practice, $4^{\mathrm{a}}$ ED. HARDCOVER. 2014.

COOPER, R. A.; OHNABE, H.; HOBSON, D. A. An Introduction to Rehabilitation Engineering (Series in Medical Physics and Biomedical Engineering). Hardcover - December 26, 2006.

FALCÃO, C.; SOARES, M. M. Usabilidade de Produtos de Consumo: uma análise dos conceitos, métodos e aplicações. Estudos em Design, Revista (online), Rio de Janeiro: v. 21, n. 2, p. 01-26, 2013, ISSN 1983-196X.

FILHO, A.N.B. Diretrizes para a escolha do produto de tecnologia assistiva. In: OLIVEIRA, A.I.A.; LOURENÇO, J.M.Q.; LOURENÇO, M.G.F. Perspectiva da Tecnologia Assistiva no Brasil: pesquisa e Prática. Belém: EDUEPA, 2008, p. $115-120$.

FINDLEY, L. The economic impact of Parkinson's disease. Parkinsonism Relat Disord. 2007; 13: 812.

FOLSTEIN MF.et al. Mini Mental state. J

Psychiat. Res. 1975; 12:189-98

GRANDJEAN, E.; KROEMER, K. H. E. Manual de ergonomia: Adaptando o Trabalho ao Homem. Posto Alegre: Bookman, 2005.

HOHMANN, P.; CASSAPIAN, M. R. Adaptações de baixo custo: uma revisão de literatura da utilização por terapeutas ocupacionais brasileiros. Rev. Ter. Ocup. Univ. São Paulo, v. 22, n. 1, p. 10-18, jan./abr. 2011.

IIDA, I. Ergonomia: projeto e produção. 2. ed. São Paulo: Edgard Blücher, 2005.

ISO 9241. Ergonomic requirements for office work with visual display terminals (VDTs) guidance on usability. Genebra, 1998.

JANKOVIC, J. Parkinson's disease: clinical features and diagnosis. Journal of Neurology, Neurosurgery \& Psychiatry, v. 79, n. 4, p. 368-
376, jul. 2007.

JORDAN, P. W. An introduction to usability. London: Taylor \& Francis, 1998.

LEVENTHAL, L. M.; BARNES, J. A. Usability Engineering: process, products and examples. Pearson, 2008.

LIKERT, R. A technique for the measurement of attitudes. Archives of psychology, vol. 22, 140, 1932.

Martignoni E, Franchignoni F, Pasetti C, Gerriero G, Picco D. Psychometric properties of the unified Parkinson's disease rating scale and of the short Parkinson's evaluation scale. Neurol Sci. 2003;24:190-1

NIELSEN, J. Usability engineering. San Diego (CA): Academic Press, 1993.

NIELSEN, J.; MACK, R. L. Usability inspection methods. New York: John Willey \& Sons, 1994.

OLIVEIRA, L. M. B. Secretaria de Direitos Humanos da Presidência da República (SDH/PR) / Secretaria Nacional de Promoção dos Direitos da Pessoa com Deficiência (SNPD) / CoordenaçãoGeral do Sistema de Informações sobre a Pessoa com Deficiência. Cartilha do Censo 2010 Pessoas com Deficiência. Brasília: SDHPR/SNPD, 2012.

PELOSI, M.B. O papel do terapeuta ocupacional na Tecnologia Assistiva. Caderno de Ter. Ocup. da UFSCar, v. 13, n 1, p. 39-46, mai./ago. 2005.

POLLOCK, N.; McCOLL, M. A.; CARSWELL, A. Medida de performance ocupacional canadense. In: SUMSION, T. Pratica baseada no cliente na terapia ocupacional: guia para implementação. São Paulo: Roca, 2003.

ROGERS, J.; HOLM, M. Avaliação das áreas de desempenho ocupacional. Seção I. In: NEISTADT, M.E.; CREPEAU, E.B. Terapia Ocupacional. Willard \& Spackman. 9. ed. Rio de Janeiro: Guanabara Koogan, 2002. p. 167-188.
Realização:

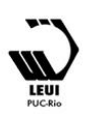




\section{$16^{\circ}$ \\ ERGODESIGN USIHC CINAHPA}

$16^{\circ}$ Ergodesign - Congresso Internacional de Ergonomia e Usabilidade de Interfaces Humano Tecnológica: Produto, Informações Ambientes Construídos e Transporte

$16^{\circ}$ USIHC - Congresso Internacional de Ergonomia e Usabilidade de Interfaces Humano Computador

CINAHPA | 2017 - Congresso Internacional de Ambientes Hipermídia para Aprendizagem.

SANTOS, A.; RUIZ, C.; FRANCISCO, N. O Uso de Atividades em Terapia Ocupacional no Tratamento do Mal De Parkinson. In: X Encontro Latino Americano de Iniciação Científica e VI Encontro Latino Americano de Pós-Graduação. Anais Eletrônicos. Disponível em: < http://www.inicepg.univap.br/cd/INIC_2006/inic/i nic/03/INIC0001028ok.pdf >. Acesso em: 25 jan. 2016.

SILVA, F.S. et. al. Evolução da doença de Parkinson e comprometimento da qualidade de vida. Rev. Neurociênc. Maringá, v.18, n4, p.46368, 2010.

SOUZA, C. et al. A doença de Parkinson e o processo de envelhecimento motor: Uma revisão de literatura. Rev. Neurocienc. v. 19, n. 4, p. 718723, 2011.

SOUZA, R.; BORGES, V.; SILVA, S.; FERRAZ, H. Quality Of Life Scale In Parkinson's disease. PDQ-39 - (Brazilian Portuguese version) to assess patients with and without levodopa motor fluctuation. Arq Neuropsiquiatr. 2007; 65:787791.

\section{Agradecimentos}

Ao CNPq pelo auxílio financeiro que possibilitou a realização desse trabalho.

Às terapeutas ocupacionais, Priscila Caldas e Juliana Amâncio, pela participação na coleta dos dados.

Aos professores José Ângelo Costa, José Junio Urbano e Jacek Michalewicz, do Curso de Mecânica do Instituto Federal de Educação, Ciência e Tecnologia de Pernambuco (IFPE), pela parceria na pesquisa e colaboração no desenvolvimento das adaptações impressas em 3D. 